\title{
Arc routing for rural Irish networks
}

\author{
P. Keenan
}

University College Dublin

Department of Management Information Systems, Belfield, Dublin 4, Ireland. Tel : +3531 706-8130.

e-mail: pkeenane irlearn.ucd.ie

\author{
M. Naughton \\ University Software Systems \\ 3 Terminus Mills, Clonskeagh, Dublin 14, Ireland.
}

\begin{abstract}
This paper discusses the capacitated arc routing problem in the context of large sparse networks drawn from sections of the Irish rural road network. The particular features of this form of arc routing are discussed. Various arc routing techniques are reviewed in this context. Two heuristics, a route first/cluster second heuristic and a clustering heuristic based on shortest path trees are proposed. These heuristics are shown to provide efficient routes and the clustering heuristic to provide routes which could form the basis of routes for practical use.
\end{abstract}

Keywords

Arc routing, heuristic, Chinese Postman

\section{$1 \quad$ ARC ROUTING PROBLEMS}

Arc Routing Problems are an important class of problems with many real world applications. This class of problems is defined on a graph $G=(V, A)$ where $V$ is the vertex set and $A$ is the arc set. Associated with the graph $G$ is a non negative cost matrix $C_{i j}$ giving the weight of the arc from vertex $i$ to vertex $j$. The simplest form of arc routing problem is the Chinese Postman Problem(CPP). The CPP involves finding a minimum cost cycle that traverses each arc in $A$ at least once. If in a graph $G$ a tour exists which traverses all edges exactly once then the graph is Eulerian. A single tour which traverses such a graph is an Euler tour. The fundamental condition for an Eulerian graph is that every vertex be of even degree. If this is not the case a minimum cost perfect matching algorithm (MCPM) can be used to add additional arcs between vertices of odd degree. For undirected graphs, the CPP can be solved in polynomial time. A number of variations on the basic CPP exist, a recent review of work in this area can be found in Eiselt, Gendreau and Laporte (1995). 
The rural postman problem (RPP) is an extension of the CPP to a case where it is only necessary to service a subset of the arcs in a network. The capacitated arc routing problem (CARP) is an extension of the CPP to a situation where a number of vehicles of limited capacity are used to service the arc network. Each arc has a non-negative weight $q_{i j}$ and each vehicle a capacity of $W$. The restriction on vehicle capacity may be distance travelled, duration of route or quantity of goods carried. Arc routing problems may be considered for cases where the arcs are directed or undirected, or a mixture of both. The RPP and CARP are reviewed in Eiselt, Gendreau and Laporte (1994).

As capacitated arc problems are NP hard a variety of heuristics have been developed. While the objective of a CARP optimisation is usually to minimise distance there are a number of other objectives that may be present in a practical problem. These include balancing the workload on different routes, not only in terms of time but also in terms of number of customers serviced or distance travelled. The shape of the routes may also be an important factor in determining customer or driver acceptance of proposed routes. Time windows may be required, these may be relatively fixed or flexible. The presence of these additional constraints has influenced the design of heuristics and has meant that, as Eiselt et al point out, research in arc routing problems is relatively fragmented. The diverse nature of arc based problems, from postal delivery to refuse collection, means that heuristics suitable in one application are often unsuitable in other contexts.

\section{IRISH RURAL ROUTING}

The problem presented in this paper is routing on Irish rural road networks, for example the delivery of letters. However the principles could also be applied to electricity meter reading, election canvassing, or other applications where all residents had to be visited.

By European standards Ireland has a substantial rural population which is dispersed over an extensive network of rural roads. The vast majority of these road segments have one or more houses. Therefore for problems such as postal delivery where every house must be visited almost all roads will have to be serviced. Since the roads to be serviced will generally form a connected network, the problem of visiting all houses in a given area will be a capacitated Chinese postman problem (CCPP). However in some sparsely populated districts the arcs to be visited might not be initially connected and a capacitated rural postman problem (CRPP) is encountered.

The nature of Irish rural road networks is such that for many problems the service time for each arc is a small proportion of the capacity of a vehicle. A postman working a seven hour day in a van could service more than 200 road segments in some cases. A typical rural area serviced by a number of vehicles from the nearest town might have more than 1000 arcs to be serviced. The rural road network gives rise to a graph that is extremely sparse compared to the networks frequently encountered in routing literature. Typically the arc/vertex ratio is between 1.1 and 1.4 .

However each road segment need only be serviced once as it is possible to service customers on both sides of the road. The rural nature of the problem means that many difficulties found in urban areas do not arise. These include problems with one way streets, no right or left turns, traffic restrictions etc. Many urban applications are based on pedestrian 
routes, these introduce problems such as delays in crossing the road (Roy and Rousseau 1989). In most urban applications both sides of the road are visited separately, effectively generating two arcs for each road in the network. Other urban applications use vehicles, but the actual service is based on walking tours around the parked vehicle. The problem then becomes a two phase one as the locations where the vehicle is to be parked must also be identified (Bodin and Levy 1991). Other arc routing problems use vehicles in applications such as refuse collection or road gritting where vehicle capacity is an important constraint (Eglese and Li 1992). In comparison to these situations, this paper address a problem which is relatively free of constraints in comparison to other real world problems. However the size of the problems encountered is larger and the networks are very sparse when compared to most examples in the literature.

\section{ALGORITHMIC APPROACHES TO THE PROBLEM}

The solution of arc routing problems has received less attention in the past than the more common node routing problems. However as for other types of NP hard vehicle routing problem, various heuristic based solution procedures have been proposed. These techniques can be grouped into those where clustering and routing takes place in a single step and those where two separate steps are used for establishing routes and the clusters to be serviced by each vehicle.

\section{Lower Bounds for CARP}

A number of lower bounds for the CARP have been proposed. These bounds require the solution of a matching algorithm on a modified graph $\mathrm{H}$ derived from $\mathrm{G}$. Early bounds were developed by Golden and Wong(1981), Assad, Pearn and Golden(1987) and Pearn(1988). The more recent bounds are those of Surawatari, Hirabayashi and Nishida (1992) and Benavent, Campos, Corberán and Mota (1992). The latter authors review all previous bounds and propose a number of improvements to them. Hirabayashi, Suruwatari, and Nishida (1992) propose a branch and bound algorithm based on their bound that provided an optimal solution for the CARP. While this algorithm allowed optimal solutions it was tested only on networks with less than 50 arcs, owing to its slow execution time. As we will demonstrate later in this paper this algorithm in its basic form is too slow for use on large Irish rural networks.

\section{Single Pass Heuristics}

A variety of single pass heuristic algorithms have been proposed. These have been tested on relatively small networks where the service time for each arc is a large proportion of the vehicle capacity. Pearn (1989 and 1991) provides a review of these algorithms. For dense graphs Pearn proposes a modification of the construct strike algorithm proposed by Christofides in 1973. This procedure is based on the repeated identification and removal of cycles from the graph with the addition of additional artificial arcs where required. Pearn found that this procedure performed well on dense graphs, but not on sparse graphs. The Irish rural networks are very much more sparse than the networks used by Pearn. In 1991 Pearn proposed augment-insert algorithms for sparse networks with high demand ratios per arc. These algorithms aimed to identify cycles where the sum of demands on the arcs in the cycle 
exceeded the capacity of the vehicle. This does not arise in the Irish rural situation where the demand on each arc is a small proportion of the capacity of the vehicle.

While these and other single pass algorithms could be modified to cater for larger and sparser networks, these modifications would largely mean the design of a completely new algorithm. We would argue that development of these existing techniques is not likely to provide effective routes in an Irish rural context.

An alternative heuristic approach is to separate the clustering and routing of the clusters. This two phase approach has provided good heuristic solutions to the related point based vehicle routing problem. The separation of routing and clustering in CARP may be even more attractive given that optimal CPP routes can be generated within each cluster. Furthermore a separate clustering procedure may allow consideration of constraints other than distance minimisation. These include the generation of 'logical' routes, where the routes use by services such as postal delivery can be justified to the public. Postal delivery problems also require the sorting of letters and this can be more easily done if adjacent addresses are on the same route.

\section{Route first, cluster second heuristics.}

These heuristics generate a large route covering the entire network, which can then be broken up into clusters. We test a heuristic based on the generation of a large Euler tour on a matched graph. Within a matched graph a number of possible Euler tours exist, we generate a large tour which passes close to the depot at the point where a vehicle's capacity would be exhausted.

\section{Cluster First Route Second Heuristics}

In point based vehicle routing problems a variety of strategies exist to cluster points before routing the clusters. In the arc routing domain a clustering algorithm has to identify a group of arcs that is likely to form a good route when the routing phase is complete. In a practical problem the clusters generated should also be acceptable, this generally entails adjacent arcs being visited on the same route. In the point based vehicle routing problem many successful procedures exist to generate routes which radiate from the depot in some type of cone shape. These group neighbouring point together and then use a travelling salesman algorithm to route within that cluster of points. A similar approach, grouping arcs, would give rise to reasonable CARP clusters. In the context of Irish rural networks we have experimented with the generation of arc based routes using shortest path trees rooted at the depot. This strategy provides radial routes which ensure that neighbouring arcs are on the same route. These clusters can be generated using the original network and within each cluster a matching algorithm can be used to generate an Euler tour.

\section{COMPUTATIONAL RESULTS}

\section{Test networks}

The networks used in this paper are derived from rural Irish road networks. Each section of the network has a travel time and a service time, which is representative of typical routing applications. In order to facilitate the operation of the algorithms a network simplification 
routine was employed to derive networks small enough to be solved optimally. This procedure eliminated unnecessary detail, including the removal of dead end roads and the inclusion of the time taken to service that arc in the section of road to which the dead end arc connects. This network simplification does not greatly affect the operation of the algorithms, but it reduces the size of the problem by up to two-thirds.

\section{Optimal solutions and lower bounds}

We attempted to find an optimal solution to the problem using the branch and bound algorithm by Hirabayashi, Suruwatari, and Nishida (1992). This algorithm proves to be unsuitable for large problems owing to the large number of sub-problems generated. We made a number of modifications to the branch and bound algorithm. We needed to modify it to take account of the fact that we used time, rather than distance, as our vehicle constraint. The most significant alteration to the operation of the algorithm was the use of an alternative branching strategy. In the initial algorithm a number of matchings are performed and the sub-problem with the highest bound selected. While this is an excellent strategy in terms of selecting a good branching arc it is computationally inefficient owing to the complexity of the MCPM algorithm, which might be solved $n / 2$ times.

An alternative strategy is to select the arc with the smallest weight as the branching arc. This alteration greatly speeds up the selection of the branching arc, although this may mean that more subproblems are generated. The original branching strategy lead to very long solution times in some cases. The use of the smallest arc as a branching strategy lead to solutions whose solution time varied much less. Therefore in some cases where the original strategy performed poorly it required solution times ten times those using the selection of the smallest arc strategy The solution time for the new branching procedure was less than half of the alternative in most cases. In some cases where the original strategy performed well the solution times were similar. A more complete discussion of these alterations may be found in Montwill and Naughton (1994). A disadvantage of the generation of an increased number of subproblems, in a practical procedure, is that the memory of the computer used is often an important limitation on the operation of this branch and bound algorithm.

In practice the branch and bound algorithm, including our amendments, remains unsuitable for large problems. A Pentium $100 \mathrm{MHz}$ microcomputer with $16 \mathrm{MB}$ of memory was used for these computations, for problems larger than 70 arcs the number of subproblems generated meant that the algorithm ran out of memory. However in all cases a lower bound was available, which provided a basis for comparison for the heuristics used. This bound, derived by Surawatari, Hirabayashi and Nishida (1992) is not the best available, but is close to it Benavent, Campos, Corberán and Mota (1992).

\section{Route First/ Cluster Second Heuristic}

This approach generates a large Euler tour, which is then split up into routes with the maximum vehicle time. Initially a matching algorithm is used to make the network Eulerian. Within this matched network a large number of possible Euler tour exists. However the large tour generated needs to be suitable for splitting into smaller tours. Therefore a number of heuristics are used when building the large tour to ensure that suitable routes result from the splitting of this large tour. 
Phase one of the building the Euler tour adds arcs, adding demand arcs where possible, generally moving in a direction away from the depot. This phase continues until approximately half of the route time of a vehicle has been allocated. In the second phase of the algorithm we attempt to keep the algorithm in the region of the most distant point routed in phase one. This attempts to ensure that routes are reasonably compact. A third phase begins when the route has completed more than $75 \%$ of the route time for a single vehicle. In this phase the large route makes it way back towards the depot. When the route time for a single vehicle is exhausted, the algorithm goes back to phase one. At the end of the routing process, when the large route has returned to the depot, checks are made to ensure that all sections of the network have been routed. If the large route returns prematurely to the depot, the addition of these unrouted arcs may affect quality of the routes generated.

This strategy should ensure that the routes are fairly compact and that the split points on the route occur close to the depot. By and large this approach provided reasonably efficient routes, but despite our fine tuning of the arc selection rules used, it proved difficult to ensure that the route stayed in the same region of the graph.

\section{Cluster First Heuristic}

This approach generated shortest path trees rooted at the depot. Sub trees whose traversal time is less than half the capacity of a vehicle are identified. Neighbouring subgroups are then combined to derive routes whose estimated travel time was close to the capacity of the vehicles. An Euler tour was then generated for each of these clusters using an MCPM algorithm. The test results present the initial clustering, further improvements are possible using an improvement algorithm to refine the boundaries of routes.

Table 150 arc network, total arc distance $15.32 \mathrm{~km}$

\begin{tabular}{ccclcc}
\hline $\begin{array}{c}\text { Vehicle } \\
\text { Capacity } \\
\text { (minutes) }\end{array}$ & $\begin{array}{c}\text { Number of } \\
\text { vehicles }\end{array}$ & $\begin{array}{c}\text { Lower Bound } \\
(\mathbf{k m})\end{array}$ & $\begin{array}{c}\text { Optimal } \\
\text { Solution }\end{array}$ & $\begin{array}{c}\text { Route First/ } \\
\text { Cluster } \\
\text { second }\end{array}$ & Cluster First \\
\hline 300 & 2 & 17.95 & 17.95 & 17.95 & 18.19 \\
240 & 2 & 17.95 & 17.95 & 19.43 & 18.19 \\
150 & 3 & 18.39 & $*$ & 21.17 & 21.86 \\
120 & 3 & 18.39 & $*$ & 20.03 & 20.98 \\
100 & 4 & 19.01 & $*$ & 23.15 & 22.57 \\
\hline
\end{tabular}

Table 265 arc network, total arc distance $17.54 \mathrm{~km}$

\begin{tabular}{llllll}
\hline $\begin{array}{c}\text { Vehicle } \\
\text { Capacity } \\
\text { (minutes) }\end{array}$ & $\begin{array}{c}\text { Number of } \\
\text { vehicles }\end{array}$ & $\begin{array}{c}\text { Lower Bound } \\
(\mathbf{k m})\end{array}$ & $\begin{array}{c}\text { Optimal } \\
\text { Solution }\end{array}$ & $\begin{array}{c}\text { Route First// } \\
\text { Cluster } \\
\text { second }\end{array}$ & Cluster First \\
\hline 360 & 2 & 22.50 & 22.50 & 24.76 & 23.28 \\
210 & 2 & 22.50 & 22.50 & 23.38 & 23.35 \\
180 & 3 & 22.50 & $*$ & 23.38 & 23.32 \\
150 & 3 & 22.50 & $*$ & 27.32 & 25.20 \\
\hline
\end{tabular}

* owing to machine limitations these problems could not be solved optimally 
Table 3 Large areas, e.g. complete postal delivery regions

\begin{tabular}{cccccc}
\hline Area & $\begin{array}{c}\text { Number of } \\
\text { arcs }\end{array}$ & $\begin{array}{c}\text { Number of } \\
\text { Vehicles }\end{array}$ & $\begin{array}{c}\text { Lower bound } \\
(\mathbf{K m})\end{array}$ & $\begin{array}{c}\text { Route first } \\
\mathbf{( K m})\end{array}$ & $\begin{array}{c}\text { Cluster first } \\
(\mathbf{K m})\end{array}$ \\
\hline 1 & 649 & 4 & 269.7 & 288.4 & 292.8 \\
2 & 600 & 3 & 307.1 & 330.2 & 322.5 \\
3 & 483 & 3 & 168.7 & 183.7 & 184.2 \\
4 & 362 & 3 & 203.8 & 224.8 & 213.8 \\
\hline
\end{tabular}

\section{CONCLUSIONS}

\section{Analysis of test results}

While the cluster first and route first heuristics have a similar performance, the cluster first heuristic provides superior routes when considerations such as geographical compactness are considered. The use of the shortest path based clustering approach provides routes which are practically useful without any great penalty in terms of distance travelled. These clusters can be derived with a negligible solution time on a modern PC, indicating their suitability for use in practical software for this problem.

This paper presents an initial examination of techniques for solving the capacitated arc routing problem on large sparse networks. These initial results indicate that the use of shortest path trees rooted at the depot can provide a good basis for clustering of routes. These clusters are not only efficient in terms of the distance of the routes provided, but also substantially satisfy the need for practical routes to be geographically compact. While other approaches may provide marginally superior solutions in terms of distance travelled, these may not ensure that routes meet the other requirements of practical routing.

\section{Further work}

The heuristic cluster could be further improved by using an Eulerian graph and explicitly taking account of the additional arc traversals needed. A variety of improvement routines could be used to swap arcs at the boundary between routes, both to improve the overall travel time and to make the routes more geographically compact. These would provide routes that were close to optimal and practically feasible, without requiring excessive program execution time.

While the branch and bound techniques tested proved too slow for practical use the possibility exists of improving these. The algorithm could make use of the superior lower bounds proposed by Benevant et al (1992). The heuristic techniques could be used to provide good initial upper bounds. Further examination of the branching strategies should lead to faster solution times. A combination of all of these might allow larger problems be solved optimally in a reasonable period of time. Even if optimality cannot be achieved, a procedure such as branch and bound could move much closer to the optimal solution in a shorter solution time. It seems likely, however, that heuristic techniques will continue to have an important role to play in solving problems on large networks. 


\section{REFERENCES}

Assad, Pearn and Golden (1987) The Capacitated Chinese Postman Problem: Lower bounds and solvable cases. American Journal of Mathematical and Management Science, 7, 63-88.

Benavent, E., Campos, V., Corberán, A. and Mota, E. (1992) The Capacitated Arc Routing Problem. Lower Bounds. Networks, 22, 669-690.

Bodin, L.D. and Levy, L. (1991) The Arc Partitioning Problem. European Journal of Operational Research, 53, 393-401.

Eglese, R.W. and Li L.Y.O. (1992) Efficient Routeing for Winter Gritting. Journal of the Operational Research Society, 43, 1031-1043.

Eiselt, Gendreau and Laporte (1995) Arc Routing Problems : part I: The Chinese Postman Problem, Operations Research, 43, 2, 231-242.

Eiselt, Gendreau and Laporte (1994), Arc Routing Problems. Part II : The Rural Postman Problem. Working Paper CRT-961, Centre de Recherche sur les Transports, Université de Montréal. (also forthcoming in Operations Research)

Golden and Wong (1981) Capacitated arc routing problems, Networks, 11, 305-315.

Hirabayashi, R., Suruwatari, Y., and Nishida, N. (1992) Tour Construction Algorithm for the Capacitated Arc Routing Problems. Asia-Pacific Journal of Operational Research, $\mathbf{9}$, 155-175.

Montwill, P. and Naughton, M. (1994) The Capacitated Chinese Chinese Postman Problem, MMangtSc disseration, University College Dublin

Pearn, W.-L. (1991) Augment-Insert Algorithms for the Capacitated Arc Routing Problem. Computers and Operations Research, 18, 189-198.

Pearn, W.-L. (1989) Approximate solutions for the Capacitated Arc Routing Problem.. Computers and Operations Research, 16, 598-600.

Pearn, W.-L. (1988) New lower bounds for the capacitated arc routing problem. Networks, 18, 181-191.

Roy, S. and Rousseau, J.-M. (1989) The Capacitated Canadian Postman Problem. INFOR, 27, 58-73.

Surawatari, Y., Hirabayashi, R. and Nishida, N. (1992) Node Duplication Lower Bounds for the Capacitated Arc Routing Problem. Journal of the Operations Research Society of Japan, 35, 2, 119-133. 